\title{
«El ser y la cuchara» \\ Meditación sobre el ser en el De Mente de Nicolás de Cusa
}

\author{
ANDRÉS HUBERT ROBINET, S.J. \\ Universidad Católica del Norte (Chile) \\ ahubert@ucn.cl
}

\begin{abstract}
Resumen
El De Mente de Nicolás de Cusa quiere ser un estudio de la mente como medida del universo y como imagen de Dios. En este libro, Nicolás se presenta a sí mismo como un Idiota o Ignorante y toma el ejemplo de la fabricación de una cuchara. La cuchara, instrumento simple, llega a ser símbolo del ser. El arte y la fabricación de este instrumento reflejan el ser humano y su esfuerzo por entender y construir el mundo (co-crear), por acercarse a Dios y a su obra de creación. La cuchara se transforma en un espejo que permite ver la realidad aunque sea de lejos; permite sobre todo acercarse al primer espejo que es Cristo.
\end{abstract}

Palabras claves: Nicolás de Cusa, ignorante, mente, cuchara, docta ignorantia, búsqueda del ser.

\section{«Being and spoon» \\ Meditation on being in De Mente \\ of Nicholas of Cusa}

\begin{abstract}
Nicholas de Cusa's De Mente pretends to be a study of the mind as the measure of the universe and the image of God. In this book, Nicolas presents himself as an idiot or ignorant and takes the example of manufacture of a spoon. The spoon, simple tool, becomes a symbol of being. The buman ability to create this tool expresses the buman nature and his effort to understand and cocreate the world, God and His work of creation. Thus, the spoon becomes a mirror that allows us to discover reality, even from afar; and moreover allows us to approach to Christ also our first or main mirror.
\end{abstract}

Key words: Nicholas of Cusa, ignorant, mind, spoon, docta ignorantia, search of the being.

Doctor en Teología de la Pontificia Universidad Católica de Chile. Director del Departamento de Teología de la Universidad Católica del Norte (Antofagasta, Chile). Autor de Palabras de Creación. La Creación en la Teología y en el método teológico de San Anselmo de Canterbury (2004).

Este trabajo está enmarcado en el proyecto Fondecyt regular 1100043 (20102011): El misterio del Ser en Hans Urs von Balthasar, Alberto Magno y Nicolás de Cusa. El misterio del ser es el origen fundante a modo del Espíritu Santo en cuanto bondad. 
En los libros del Idiota, Nicolás de Cusa pone como centro de los diálogos a un hombre que él llama «Idiota» o Ignorante'. En el De Sabiduría, éste conversa con el Orador, y en el De Mente con el Filósofo. En ambos, el mismo idiota profundiza las ideas y los temas preferidos del Cusano. En la lectura del Idiota. De Mente, salta a la vista una reflexión extraña que introduce en la meditación del ser: se trata de la descripción de un Ignorante que es artesano, fabricante de cucharas. Deberíamos llamarlo artista más que artesano. El artesano fabrica cucharas desde el modelo de las cucharas. El artista ve la cuchara en su mente y en el pedazo de madera. ¿Es solamente una reproducción de las ideas platónicas? ¿Es posible unir el ser con la cuchara?

Este ejemplo de la cuchara es único en el Corpus cusano; también es única la manera cómo lo presenta el autor. Por eso, merece que le demos nuestra atención. En este trabajo, se quiere mostrar la importancia de este ejemplo. Se avanzará por distintos pasos. En la primera parte, se resumirá el tratado Idiota. De mente para ver qué es y quién es el idiota. En una segunda parte, se profundizará la visión del artista sobre la fabricación-creación de la cuchara. Esta segunda parte será la más importante.

El método empleado consiste en analizar los textos mismos de Nicolás para acercarse lo mejor posible al pensamiento del autor y aprovechar al máximo su metafísica mística.

\section{Los actores}

\section{E1 De Mente}

Nicolás de Cusa parte de la definición y función de la mente: «La mente es desde la cual se da la medida y el término de todo» (DM 57) ${ }^{2}$. O la mente es «aquella fuerza que reside en nosotros, la cual complica (es decir, tiene implícita en sî) nocionalmente los ejemplares originales de

1 Los libros del Idiota son: Idiota. De Sabiduria (libro I y II) (en adelante, DS), Idiota. De Mente (en adelante, DM) e Idiota. De Staticis experimentis. Para los textos de Nicolás de Cusa se utilizó en este artículo la edición crítica de P. Wilpert (Kues, 1967). Aunque la traducción de los textos del Cusano es propia debo señalar que fueron de gran utilidad los trabajos de Jorge M. Machetta (ver referencias al final). Otras abreviaturas de las obras del Cusano en este artículo son las siguientes: ADI (Apología de Docta Ignorantia); DB (De Berillo); DDI (De Docta Ignorantia); DFD (De Filiatione Deı); DG (De Genesi), DVD (De Visione Dei).

2 «Mentem esse, ex qua omnium rerum terminus et mensura». 
todo» (DM 58) ${ }^{3}$. Pero al mismo tiempo, la razón no puede alcanzar la esencia de las cosas. Esto significa que no las puede nombrar (DM 59). Podemos partir de la forma que se une a la materia y le da nombre que existe desde siempre (los nombres son eternos: provienen de la eternidad). Lo mismo podemos hacer con la cuchara (DM 59).

Todo arte es imagen del arte infinito y divino. Al mismo tiempo, ningún arte ha alcanzado la perfección porque es finito y limitado (DM 60). El arte infinito es principio de todo y principio sin principio (DM 61).

Veamos la cuchara. Todo artista tiene la idea en su mente porque compara con otra realidad. El Ignorante no quiere imitar otra cuchara (ni copa u olla). Busca ser realizador más que imitador y así ser semejante al arte infinito (DM 62). ¿Cómo sacar la forma de la cuchara? Los artistas parten de los sentidos. El Ignorante quiere cavar la materia hasta que surja la cuchara (DM 63). Así, «resplandece la forma de la 'cucharidad', simple y ajena a los sentidos (insensibilem)» ${ }^{4}$. Lo propio de la cuchara es incomunicable, no puede hacerse perfectamente sensible. Se la llama «cuchara» por imposición de nombre (DM 64). En eso actúa la razón: ella estudia las cosas sensibles, busca la superación, la concordancia y la diferencia. Esto significa que la razón se queda en conjeturas y opiniones, porque no encuentra ni puede encontrar la forma. Los nombres dependen de los sentidos y desaparecen cuando las cosas desaparecen (DM 65). Entonces, el artista no debe utilizar los sentidos, sino dejar que aparezcan los ejemplares: «Los modelos originales preceden, por naturaleza a las cosas sensibles, como la verdad a la imagen $\rangle^{5}$.

Nuestra mente tiene un juicio concreado con ella (DM 77). «Concreado» significa algo que recibió por naturaleza, que nunca aprendió. Es importante entonces que este don sea excitado para ser ejercitado y desarrollado (DM 78). Para crear algo, hay que conocer el todo, hay que concebir el todo: sólo así se puede confeccionar una cuchara perfecta, bien proporcionada y proporcionada en orden al todo. Así «es necesario que a la ciencia de una única parte preceda la ciencia del todo y de sus partes» (DM 127) ${ }^{6}$. La universalidad es primera y «Dios es el modelo original de la universalidad» $(\mathrm{DM} 127)^{7}$. Lo primero es la ciencia de Dios y de lo universal.

3 «Vim illam quae in nobis est, omnium exemplaria notionaliter complicantem». Complicare es «tener implícito en sí». Lo contrario es explicare que significa «explicitar».

«Formam coclearitatis simplicem et insensibilem resplendere».

«Exemplaria natura praecedere sensibilia sicut veritas imaginem».

«Unde necesse erit, ut ad scientiam unius praecedat scientia totius et partium eius».

«Quare Deus, qui est exemplar universitatis». 
Tenemos que partir de esta primera verdad: hay que conocer a lo universal para conocer lo particular. Hay que conocer a Dios para conocer su creación. Para crear una cuchara real, hay que conocer la madera y tener la cuchara ya en la mente. Pero ¡cuidado! (y es la segunda verdad), la cuchara en la mente no existe mientras no se esculpa en la madera: «el poder ser no es» (DM 107) ${ }^{8}$. Pero el poder ser (la posibilidad) es anterior al existir (DM 130).

Tanto el poder ser hecho como el poder hacer son anteriores y, al mismo tiempo, ambos necesitan unirse para poder existir: el nexo es fundamental. Y podrá decir Nicolás que todo auto-subsistente es trinitario: tiene su esencia, su sabiduría y el nexo que une a ambos (DM 147).

Hay que partir de arriba, partir de lo universal. Hoy estamos acostumbrados a ver la ciencia partiendo de cosas o casos individuales y sacar leyes. Es el desafío y el problema del Positivismo. Para Nicolás, si queremos conocer la parte, hay que conocer el todo (DM 127). Así con la cuchara. Si se ignora a Dios, es imposible conocer la universalidad. Al mismo tiempo, la universalidad, como el ser, es algo indeterminado. La universalidad no es. Y el ser tiene el mismo peligro si se queda en lo universal: hablamos de humanidad, pero sólo conocemos a «hombres» (DM 140).

Si vemos las cosas antes que comiencen a ser, sólo hay eternidad (DM 129). La universalidad existe desde la eternidad (DM 130). Así hay seguridad que la conexión exista antes que la división. Y el nexo es el Espíritu Santo.

La universalidad es posibilidad y el poder ser no es (DM 138). ¿Cómo, entonces, ver la realidad? El número ve la pluralidad (DM 143). Pero en la mente divina todo se une porque la Trinidad une (138). Si se ve desde abajo (como lo hace Aristóteles), tenemos la naturaleza que actúa por necesidad absoluta y Dios llega a ser sólo el arquitecto (DM 151-153). Si se mira desde arriba, entonces la ciencia es ver que se cumpla y cómo se cumple la voluntad del amo (Lc 12,47-48) para que Dios sea todo en todos (1 Co 15,28) (DM 145). No podemos olvidar que, en Dios, el querer coincide con el ejecutar (Ez 37,14). No podemos olvidar tampoco que Dios es imparticipable porque es simple (DM 153).

Este resumen es parcial, pero tiene el valor de presentar los temas relevantes de Nicolás de Cusa: el ser y su relación con el creador. El ser es trinitario. Por eso la importancia de la Trinidad en la obra del Cusano (especialmente en el De Docta Ignorantia y en el De Visione Dei). También esto es fundamental para entender lo que es en sí la Docta Ignorancia.

8 «Posse esse non sit». También: «possibile esse non sit» (DM 138). 
Los otros temas importantes son: la búsqueda de los nombres (o de la esencia) de las cosas, la relación entre lo uno y lo múltiple, y, lo que será importante en este trabajo, la primacía absoluta de Dios en el entendimiento de todo el universo. Este trabajo busca profundizar la idea de ser. Para eso, tendremos que partir con el primer ente encontrado: el idiota.

\section{E1 Idiota ${ }^{9}$}

Partiremos por la presentación del personaje principal: el idiota. Nicolás nunca lo define. Para conocerlo, tenemos que avanzar a tientas. Lo único seguro es que no podemos quedarnos con el sentido moderno del vocablo que refiere a una enfermedad psiquiátrica o al desprecio de una persona.

El término griego «idiota» significa lo propio y el vocablo «idiotés» llegó a significar el nombre propio de una persona. En latín, «idiota» o «idiotés» recuerda a un hombre sin profesión, a un ignorante. Por eso, la traducción de este término se rinde con «ignorante». Pero en el latín eclesial de la Edad Media, tuvo una relevancia especial. La Iglesia se divide entre «monjes, cleros, los espirituales y los laicos, los carnales, los que están comprometidos con las realidades mundanas... El concepto de litteratus se reserva para el clero y el de illitteratus o idiotas para los laicos» (Silva, 2010: 288). El idiota es entonces una persona no letrada, sin estudios. Pero si remontamos más atrás todavía, debemos recordar que, entre los primeros cristianos «No hay muchos sabios según la carne ni muchos poderosos ni muchos de la nobleza... Lo plebeyo y despreciable del mundo ha escogió Dios» (1 Co 1,26-28). Los apóstoles eran conocidos como «hombres sin instrucción ni cultura» (Hch 4,13) ${ }^{10}$. Agustín insistirá en este aspecto. Recordará los textos de San Pablo a los Corintios para avisar que el Señor «eligió primero a hombres sin cultura» (o sea, idiotas) ${ }^{11}$, y después de la Ascensión, «envió al Espíritu Santo que hizo que sus discípulos, hombres sin cultura (idiotas), pudieran hablar las lenguas de todos los pueblos» ${ }^{12}$.

Podemos tomar un ejemplo de la Edad Media (Matteoli, 2010). En una carta a toda la Orden franciscana, San Francisco se acusa: «He

\footnotetext{
9 Para este apartado, nos hemos servido de la presentación de González Ríos (2005).

10 El texto griego pone: $\alpha \gamma \varrho \alpha \mu \mu \alpha \tau o \iota n \alpha \iota \iota \delta \iota \omega \tau \alpha \iota$, que la Vulgata tradujo por: sine litteris et idiotae.

11 En In Psal. 65,4: «quos primum idiotas elegit».

12 En In Psal. 96,2: «misso Spiritu Sancto fecit discípulos suos, idiotas homines, loqui omnium gentium linguis».
} 
pecado mucho por mi culpa, especialmente porque no he observado la Regla que prometí al Señor, ni he dicho el Oficio como la Regla prescribe, tanto por negligencia como a causa de mi enfermedad». Y añade dos motivos: «ser ignorante e idiota». Estos dos términos deben diferenciarse y, además, ¿cómo ver una culpa en ellos? ¿En qué podemos nosotros ver algo culpable en el Pobrello? Lo de ignorante, hay que entenderlo en que, en una orden creciente, que se ha convertido en una orden clerical (es decir, con estudios), Francisco se acusa de no haber buscado la adquisición de una cultura notable (es ignorante). Además se acusa de ser idiota. Esto debe entenderse en el sentido que Francisco prefiere una vida retirada, carente de autoridad. Francisco se explica recordando que, en los comienzos, los primeros hermanos tenían una vida de oración, sumisión, trabajo. «Decíamos el oficio, los clérigos con los clérigos, los laicos decían los Pater Noster... Éramos idiotas y súbditos de todos». «Idiotas y súbditos de todos» es la descripción de la vida franciscana en sus inicios: hay simplicidad, sobriedad en la falta de posesiones materiales (iglesias propias, por ejemplo) o culturales. Además todos estaban a disposición de todos. El «idiota» se puede traducir como «una persona privada de autoridad cultural, privada de cualquier título».

A esta definición, el Cusano añade que el idiota es pobre (DS I, 1). Varias veces el mismo protagonista repetirá que es ignorante y sabemos que, para Nicolás de Cusa, la ignorancia es el comienzo de la sabiduría porque llega a ser docta. Es el origen del pensamiento de Nicolás que quedó plasmado en su primer gran libro: La Docta ignorancia.

En este sentido, el Idiota es el mismo Nicolás de Cusa. Es evidente que el Cardenal tiene su título y su biblioteca, pero ha conservado el deseo de leer el libro principal que es la creación misma. No podemos olvidar que la sabiduría grita fuera, en las plazas, y su clamor resuena porque habita en las regiones altísimas (al respecto, DS I, 3, cita a $\mathrm{Pr}$ 1,20; en este sentido, podemos también recordar el Sal 19,2-5).

Su manera de enfrentar la sabiduría lo hace humilde, al contrario del orador que, creyéndose sabio, pierda su sabiduría. La ciencia sólo se logra en la humildad: ésta transforma la ignorancia en «docta ignorancia» (DS I, 4). Según la sentencia, el ignorante no sólo no tiene estudio, sino que no puede llegar a la ciencia (DS I, 4).

Nicolás nos presenta entonces a un idiota o ignorante, que es iletrado con respecto a los libros escritos, pero no con respecto a los libros escritos por el dedo de Dios, escritos en todas partes en la naturaleza. Frente al Ignorante están el orador (DS) que se dedica «al 
estudio de las letras sin el cual nadie saca provecho» ${ }^{13}$ y el filósofo (DM) que «se reconoce por la palidez de su rostro, por su toga larga y por los demás signos, que muestran la seriedad de un varón reflexivo» (DM $51)^{14}$. El filósofo es un hombre letrado que sabe la importancia de las letras y de su aprendizaje. Su rasgo principal es la seriedad. Su trabajo es buscar, investigar, conversar con otros sabios, razonar (DM 52). Frente a ellos, el Ignorante-Nicolás profeso «un camino de conocimiento filosófico hacia la verdad (que es inalcanzable) en el que la filosofía y los principios de la razón deben ser superados por la metodología de la idiotez» (González Ríos, 2005: 154).

El filósofo es peripatético (DM 53). Quizás lo importante (y esto lo salva) es que sabe admirar y la admiración lleva al deseo de saber (DM 51; DDI I,1,1). Su primera admiración concierne la fe de las multitudes: «La fe es de todos y es una dentro de la diversidad de cuerpos» (DM 51) ${ }^{15}$. Cada creyente es distinto y todos vienen de partes del mundo tan alejadas y, sin embargo, la fe es la misma. El problema de la unión entre diversidad y unidad se presenta también a nivel de la fe. Además el filósofo deberá aceptar que «Los ignorantes alcanzan con la fe mayor claridad que los filósofos con la razón» (DM 52) ${ }^{16}$. La razón, con todos sus esfuerzos de investigación no alcanza lo que busca «tan perfectamente y con clara razón, como este pueblo ignorante con la fe» (DM 52) ${ }^{17}$. Estamos de nuevo en la descripción del ignorante que ya hicimos y podemos recordar la iluminación de Jesús: Dios oculta los misterios del reino a los sabios e inteligentes y las revela a los pequeños. Así es su beneplácito (Mt 11,25-26). Estamos también en el Credo ut intelligam agustiniano o anselmiano. La fe es primera porque toda visión y toda inteligencia viene de Dios. Además la fe porque es primera, ayuda a dar claridad y seguridad en la búsqueda racional. Esto no impide al filósofo (Nicolás u otro) seguir investigando. Pero hay que entender su esfuerzo como «la fe que busca inteligencia» (fides quaerens intellectum). Esta pequeña descripción del filósofo nos muestra el marco de referencia donde se sitúa el Cusano.

Sabemos también que el idiota es artesano: sabe fabricar cucharas, platos y ollas. Pero él mismo prefiere llamarse artista. Nuevamente, el

\footnotetext{
13 DS I, 1: «studium litterarum, sine quo nemo proficit».

14 «Ex faciei pallore, toga talari et ceteris cogitabundi viri gravitatem praesignantibus cognoscens». La toga talar era una vestimenta que llegaba hasta los pies, por eso la traducción (personal) de «toga larga».

15 «Omnium fidem unam in tanta corporum diversitate». Nicolás de Cusa volverá sobre este tema en su De Pace Fidei.

16 «Idiotas clarius fide attingere quam philosophos ratione».

17 «Perfecte ac lucida ratione attigi quemadmodum hic ignorans populus fide».
} 
cardenal-idiota se presenta. No conocemos su veta artística, pero está claro que aprecia las artes en general y sabe admirar, quedarse en contemplación ante una pintura de R. van der Weyden y así escribir un libro místico, el De Visione Dei. El ignorante gusta el utilizar ejemplos sacados de la matemática, al igual que el Cardenal y sus tratados matemáticos.

Tenemos que añadir que el ignorante además de iletrado, es fabricante de cucharas, vive en un subterráneo (DM 54). Con todo esto, Nicolás nos indica la pobreza y humildad de aquel hombre frente a la soberbia del Orador (DS) y a la seriedad grave del Filósofo (DM). Frente a cada uno, el ignorante vive y se siente plenamente libre. Esa es su mayor fuerza: no se siente preso de alguna escuela filosófica o gramatical, dice lo que siente, de manera libre, sin ponerse grave y sin temor a caer como los filósofos letrados (DM 55); siente placer (libenter) en su trabajo y en la conversación; su trabajo, para él, es un arte; prefiere partir de la experiencia y no de los libros (DM 55); recibe en sus despachos a personas importantes con respeto, pero sin las mínimas comodidades (DM 56). No acepta pertenecer a alguna escuela filosófica ni acoge autoridad de ninguna (DM 88). En fin, acepta para sí el nombre de «ignorante» (DM 57).

Volvamos a nuestra pregunta: ¿Cómo traducir con exactitud el vocablo «idiota»? Cada traducción será imprecisa. «Profano» o laico no tiene mucho sentido en este libro. «Idiota» es una palabra dura para el oído moderno ${ }^{18}$. «Ignorante» no refleja suficientemente la profundidad del pensamiento cusano. El vocablo «iletrado» se acerca más a la idea del autor, pero es difícil llamar a una persona «iletrado» aunque sea para un recurso literario. Para facilidad del discurso, parece preferible utilizar el vocablo «ignorante». Pero, esta traducción se vuelve más difícil cuando el mismo ignorante se confiesa «ignorante sin letras» (Ignorantem idiotam) ${ }^{19}$. Hay que repetir que la traducción «Ignorante» no es quizás la más adecuada, pero refleja las ideas propuestas y además recuerda la importancia de la Docta Ignorancia. Esta es la traducción que se utilizará en este trabajo.

18 En las traducciones francesas, M. de Gandillac (en 1942) traduce «idiota» por profane (profano) y J. F. Coursaget (en 2009) opta por idiot, porque «ce qu'il y a de plus humble, de plus pauvre, de plus modeste et de plus faible dans la hiérarchie humaine. En quelque sorte un minimum absolu, ou presque, le plus opposé assurément à ce maximum absolu, ou presque, que représente l'Orateur ou le prélat» (citado por Gress, 2010). En inglés, Hopkins prefiere hablar de layman (lego o laico).

19 J. Machetta traduce por «rudo e ignorante». Cfr. DS I,1: «pauper idiota et penitus ignorans». 


\section{La cuchara}

\section{El encuentro}

El ignorante es fabricante de cucharas. Es tiempo de preguntarnos qué es una cuchara y qué rol tiene en el libro y en el pensamiento del Cusano.

La fuerza del Ignorante es que es «idiota» e iletrado: no sabe explicar ideas a través del lenguaje. Pero es experto en escuchar «la sabiduría que clama en las calles y espacios públicos» y sabe que «este clamor existe porque ella habita en lo más alto» (DS I, 3$)^{20}$. El orador y el filósofo están presos de sus ideas y de sus libros. Se parecen a un caballo encerrado en un establo: todos ellos «se nutren con un alimento que no les es propio ni natural» (DS I, 2) ${ }^{21}$. Este alimento les quita libertad, es signo de su esclavitud. Les falta abrirse y escuchar el grito de la sabiduría. Por eso, «es un don de Dios que los ignorantes llegan a la fe con mayor claridad que los filósofos a la razón» (DM 52) 22 . Los ignorantes saben recibir la sabiduría como don de Dios. Este don corre por las calles. Hay que recogerlo primero, para poder reflexionar después.

Además, el ignorante es artista y artesano. Desde Platón (que gustaba de pintar) (DM 54), el artista tiene en la mente la idea de lo que va a expresar en su pintura o escultura. Para el idiota no es suficiente. En primer lugar, es cierto que:

...el artista que esculpe una estatua en la piedra, ya tiene en sí la forma de la estatua, como una idea. Con ciertos instrumentos que él mueve, la forma misma de la estatua produce la representación e imagen de esas ideas (DDI II, 10,151$)^{23}$.

El movimiento de los instrumentos se asemeja al movimiento de la conexión entre la potencia y el acto (DDI II, 10,151-152).

Los antiguos estoicos decían que todas las formas están en acto en la posibilidad. Allí se esconden y aparecen cuando se levanta lo que las cubre.

\footnotetext{
20 «Sapientia foris clamat in plateis, et est clamor eius, quoniam ipsa habitat in altissimis».

21 «Pascitur enim... pabulo alieno et non naturali».

22 «Dei donum esse idiotas clarius fide attingere quam philosophos ratione». Cfr. Mt, 11,25-26.

23 «Artifex vult statuam in lapide exculpere formam statue in se habens quasi ydeam per quedam instrumenta que movet ipsan formam statue in figura ydee et in eius ymagine efficit».
} 
Así la cuchara se hace de madera si se quita solamente unos pedazos (DDI II, 8,134$)^{24}$.

Nicolás recuerda la Teología Mística del Pseudo Dionisio e muestra que los peripatéticos insisten en una producción (efficere) (DDI II, 8,135). Pero hay algo más. Entramos en lo más importante del diálogo.

En el texto, el orador alaba al ignorante delante del filósofo (DM 53) y lo encuentra en un cierto local subterráneo. Allí «habla al ignorante que estaba exprimiendo (exprimere) una cuchara desde la madera» (DM 54) ${ }^{25}$. No se trata de la práctica platónica de expresar en la materia la cuchara ideal, sino, literalmente, de exprimir la cuchara. Ésta está en la madera: hay que exprimirla. Veremos más adelante la diferencia entre «fabricar»y «exprimir» y la importancia de esto último.

El encuentro entre el ignorante y el filósofo se hace bajo buenos auspicios. El orador los presenta unos a otros, pero parece despreciar al ignorante: siente vergüenza (erubeo) por las obras toscas (rusticis operibus) que ejecuta. Sin embargo, para el ignorante, la fábrica de cuchara es un arte y, como arte, nutre (pascere) la mente y el cuerpo. El filósofo se siente acogido y recuerda que Platón pintaba. Desde el primer encuentro de los protagonistas, tenemos una comparación entre Platón y el ignorante. La cuchara es el punto de unión entre ambos: el arte ayuda a la especulación.

En nuestro mundo actual, la pintura sigue siendo arte, el hacer cuchara no. Y si alguien hiciera alguna cuchara, se le llamaría artesano, y no artista. Ambas palabras empiezan con «arte», nacen del arte, pero sólo el artista se reconoce como el que crea arte; el artesano sólo se ve como el que fabrica cosas. Signos del tiempo: las pequeñas profesiones (confeccionar cucharas, ollas, platos, crear herramientas especiales, para la ebanistería, por ejemplo) ya no existen o fueron absorbidas por las grandes industrias. No es queja. Es simplemente insistencia para recalcar la vergüenza del Orador que parece rebajarse al mostrar al Filósofo las «obras toscas» (operae rusticae) del Ignorante. Así se resalta la importancia del Ignorante y de su admiración por una 'simple' cuchara, admiración que él quiere sea nuestra.

24 «Unde aiebant veteres stoyci formas omnes in possibilitate actu esse: sed latitare et per sublationem tegumenti apparere, quemadmodum si coclear ex ligno fit per ablationam partium tantum». Tenemos la única referencia de la cuchara fuera del $D e$ Mente.

25 «Idiotam ex ligno coclear exprimentem alloquitur». Esta traducción se aleja de la que ofrece J. Machetta («habló al ignorante que fabricaba una cuchara de madera»). 
El Ignorante presenta su trabajo: «Me dedico con gusto en estos ejercicios que nutren incansablemente mente y cuerpo» (DM 54) ${ }^{26}$ : alegría y gozo del artista que sabe que su arte es fuente de alimento físico y mental. El mismo Platón, porque pintaba, no veía contradicción entre arte y especulación. Estas obras toscas que avergonzaban al Orador son fuente de gozo para el Ignorante, son causas de alimentación (pascere) para la persona entera.

Puede entonces describir con efusión su temática y su manera de trabajar:

Aquí está mi arte: a través de símbolos investigo lo que quiero, alimento la mente, cambio por completo las cucharas y reanimo mi cuerpo. Así alcanzo todo lo que me es necesario y me basta (DM 55) ${ }^{27}$.

Y anuncia que trabaja con tres finalidades: la primera es mental, la segunda artística y la tercera corporal: eso es lo necesario. Las cucharas están en la parte artística.

Profundicemos esta descripción que hace el Ignorante de su arte. El Ignorante-Nicolás utiliza cada palabra con cuidado y le da importancia. Habla de investigar (inquiro), de símbolos (symbolice) y también de libertad (id quod volo). Acota que alimenta su mente con el verbo «depascere» que recuerda el apacentar el rebaño. Describe su trabajo como un cambiar, alterar (commuto) las cucharas. La idea del autor es mostrar aquí que no tiene un modelo fijo de cucharas, sino que cada cuchara, fruto de su arte, es única: ninguna es igual a otra. Por fin, con este trabajo, puede reanimar (reficio) el cuerpo. El trabajo permite comprar alimento, pero lo más importante es que este mismo trabajo es un aliciente para su vida humana. En el Idiota. De Sabiduría, Nicolás habla de pregustación (praegustatio). Nuestra vida es deseo de la sabiduría y felicidad y entonces es pregustación de la sabiduría eterna. Si uno se dirige hacia ella, descubre que la sabiduría eterna es causa del deleite. Nicolás toma el ejemplo del imán que atrae el fierro, aunque éste sea más pesado: el fierro posee una pregustación del imán (DS I, 16). Así nuestro espíritu tiene su principio en la sabiduría eterna y busca ser admitido en la dulzura pregustada (DS I, 17). El gusto es fundamental y el gusto se queda vacío y estéril si no tiene alguna experiencia sensible. No se puede gustar de oído; sólo por experiencia. No bastan las descripciones del gusto, hay que hacerlo suyo. Así cuando alguien encuentra un tesoro,

26 «Ego in his exercitiis libenter versos, quae et mentem et corpus indesinenter pascunt».

27 «Immo in hac arte id quod volo, symbolice inquiro et mentem depasco, commuto coclearia et corpus reficio: ita quidem omnia mihi necessaria, quantum sufficit, attingo». 
compra el campo (Mt 13,44) y vende todo lo que tiene (Mt 19,21) (DS I, 19). Por eso, es bueno recordar que la mente necesita del cuerpo (DM 77) y, gracias al cuerpo, está ligada al mundo (DM 73). «Cuando el hombre conoce participa su naturaleza de imagen a todos los demás aspectos del universo que carecen de mente» (Rienzo, 2005: 167). El hombre concentra dentro de sí todo el universo y, por eso, es reconocido como un microcosmos, es decir, un pequeño mundo ${ }^{28}$. Así muestra su unidad con el universo creado. La mente es semilla divina (DM 81) y debe ser no sólo «mera» imagen, sino imagen «viva» porque recibe y da vida.

Y resume Nicolás (DM 55): así tengo todo lo que necesita. Tenemos los tres estados: mente, arte, cuerpo. De la mente ya hablamos: la mente mide y es imagen de Dios. El cuerpo o lo corporal parece evidente: es la vida que cae bajo los sentidos. Preguntémonos: ¿qué es el arte? La mente permite investigar a través de símbolos (symbolice). Lo «simbólicamente» es, según la Docta Ignorancia, la manera de investigar lo invisible a través de lo visible y esto muestra la unidad profunda de todas las cosas (DDI I, 11, 30). Hay una vocación sacramental en el universo que muestra su unidad y, por ende, el valor y la unicidad del Creador.

\section{E1 arte}

¿Cómo entender el arte? Nicolás ciertamente tiene una formación artística y se relacionó con los artistas de su época, pero no dedicó ningún trabajo para profundizar el sentido del arte. Hay que entender entonces que «la estética es para él, más una tonalidad característica de todo su discurso que propiamente un tema autónomo de su actividad reflexiva» (André, 1995: 550).

Volvamos otra vez a la Docta Ignorancia. La segunda parte del libro empieza y termina con esta idea, en una especie de inclusión (D'Amico, 2005: 22-23). Nicolás habla del arte de calcular (DDI II, 1,91). Para él como matemático, es importante. Al mismo tiempo, indica que el ser humano carece de precisión para estudiar los astros, insiste en que es

28 Cfr. DDI III, 3, 198: «Humana vero natura est illa que est supra omnia Dei opera elevata, et paulominus angelis minorata intellectualem et sensibilem naturans complicans, ac universa intra se constringens ut microcosmos aut parvus mundus a veteribus rationabiliter vocitetus» (La naturaleza humana es aquella que está elevada por encima de todas las obras de Dios y es apenas inferior a los ángeles (Sal 8,6). Ella tiene implícita en sí la naturaleza intelectual y sensible y afianza dentro de sí al conjunto de la creación. Por eso fue llamada con razón microcosmos, o sea pequeño mundo, por los antiguos). 
imposible la igualdad en geometría, en música y en aritmética, lo mismo en el arte (DDI II, 1,92-94).

Cuando alguien procurase durante mil años imitar a otro en cualquier aspecto, sin embargo nunca alcanzaría la precisión a pesar de que en algún modo, la diferencia sensible no sea perceptible. El arte imita a la naturaleza en cuanto puede, pero jamás podrá llegar a la precisión de la misma (DDI II, 1,94$)^{29}$.

Al final del segundo libro de la Docta Ignorancia, Nicolás discurre nuevamente sobre el arte. Cuando admiramos la creación, nos elevamos hasta el arte y la ciencia de Dios. Porque Dios utilizó las artes en su obra creadora. Por eso podemos entender y estudiar los cuatro elementos (DDI II,13,175). Dios creó todo según número, peso y medida (Sab 11,20). El número recuerda la aritmética, el peso la música, la medida la geometría (DDI II,13,176). Así entendemos que cada elemento contenga alguna semejanza con Dios (DDI II,13,177). Todo nos lleva hacia la astronomía, es decir la visión y comprensión del universo (DDI II,13,178). Admiramos las obras, pero no sabemos la razón (DDI II,13,179).

Para Nicolás, el arte imita la naturaleza. Esto significa que la naturaleza es primera y que el arte «imita», pero no logra nunca reproducir la naturaleza en su exactitud. Ars imitatur naturam, es un adagio que viene de Aristóteles. Santo Tomás lo retoma en In octo libros physicorum Aristotelis expotitio (L. 2, 1. 4, $\mathrm{n}^{\circ}$ 5) y habla de ciencia natural y ciencia artificial. Esto ocurre porque

...el principio de la acción artificial es el conocimiento. Todo nuestro conocimiento viene a través de los sentidos desde las cosas sensibles y naturales y actuamos por semejanza de las cosas naturales en las artificiales. Vemos que las cosas naturales son imitables a través del arte porque desde un principio intelectivo, toda naturaleza está ordenada a su fin y así se ve que la obra de la naturaleza es obra de una inteligencia y avanza hacia fines seguros por unos medios determinados. Entonces cuando actúa, el arte imita (L. 2, 1. 4, n $\left.{ }^{\circ} 6\right)^{30}$.

29 «Etiam si mille annis unus alium imitari studeret in quocunque, nunquam tamen precisionem attingeret, licet differentia sensibilis aliquando non participetur. Ars etiam naturam imitatur quantum potest, sed nunquam ad ipsius precisionem poterit pervenire» (trad. de J. Machetta). Notemos el «Ars naturam imitatur» sobre el cual volveremos.

30 «...principium operationis artificialis cognitio est; omnis autem nostra cognitio est per sensus a rebús sensibilibus et naturalibus acepta: unde ad similitudinem rerum naturalium in artificialibus operamur. Ideo autem res naturales imitabiles sunt per 
Para expresarse mejor, Tomás añade: el arte imita la naturaleza en cuanto puede (in quantum potest). Nicolás sigue su ejemplo: «El arte imita la naturaleza en cuanto puede, pero nunca podrá alcanzar la precisión de la misma naturaleza» (DDI II,1,94) ${ }^{31}$.

Entendamos bien: con mayor profundidad que nosotros, los antiguos y medievales mantenían una relación armoniosa con la naturaleza. Los cristianos medievales fundamentaban su reflexión en los dos libros de la Revelación: el libro de la Escritura y el libro de la Naturaleza o Creación. Dios se nos revela en la palabra escrita (que es palabra de Dios) y, en la creación, el hombre encuentra presencia y huellas del Creador. Por ejemplo, San Buenaventura veía el libro de la creación como principio eficiente y el libro de la Escritura como principio de restauración ${ }^{32}$. Ya San Pablo criticaba a los que «habiendo conocido a Dios, no le glorificaron como a Dios ni le dieron gracias, porque lo invisible de Dios, desde la creación del mundo, se deja ver a la inteligencia a través de sus obras: su poder eterno y su divinidad» $(\mathrm{Rm}$ 1,20-21). La creación muestra el poder de Dios y, al mismo tiempo, canta y alaba su grandeza. El artista se adentra en la creación para encontrar allí las muestras claras (vestigia) de la presencia y de la acción del Creador.

El defecto del Orador es que se aleja de lo sensible y confía en argumentación y en sus lecturas (DS I,27). El defecto del Filósofo es creer que todo sale de los libros: amontona textos y se angustia cuando pregunta cómo entenderlos (DM 55). Teme caer y se pone muy serio. Entre ellos, el ignorante «se ve empujado con mayor facilidad a decir las cosas que siente» (DM 55) ${ }^{33}$. Es cierto que, en general, «los ignorantes alcanzan mayor claridad por la fe que los filósofos con la razón» (DM $52)^{34}$.

La primera revelación es darse cuenta, acoger la creación, la realidad. La sabiduría no está primero en los libros ni en las autoridades: «La

artem, quia ab aliquo principio intellectivo tota natura ordinatur ad finem suum, ut sic opus naturae videatur esse opus intelligentiae, dum per determinata media ad certos fines procedit: quod etiam in operando ars imitatur», cfr. André, 1995: 562.

31 «Ars etiam naturam imitatur quantum potest, sed nunquam ad ipsius precisionem poterit pervernire».

32 «Ratio autem ad intelligentiam praedictorum haec est: quia, cum primum principium reddat se nobis cognoscibile et per Scripturam et per creaturam, per librum creaturae se manifestat ut principium effectivum, per librum Scripturae ut principium reparativum» (Breviloquium, Pars II, c. 5,2: La razón para entender lo dicho antes es la siguiente: cuando el primer principio se nos da como cognoscible tanto por la escritura como por la creación, se manifiesta por el libro de la creación como principio eficiente y por el libro de la Escritura como principio de restauración).

33 «Arbitror neminem facilius me cogi posse, ut dicat quae sentit».

34 «Idiotas clarius fide attingere quam philosophos ratione». 
sabiduría grita en los lugares públicos y allí está su clamor porque ella habita en lo más alto» (DS I,3). El ignorante está atento, sabe escuchar este clamor. Aprendió a unir la gracias al Padre por haber revelado todo a los pequeños (Mt 11,25) con la necedad de la sabiduría humana (1 Co 1, 19-25). El hombre abierto a la revelación natural come un alimento natural (DS I,2-3) y puede encontrar a Dios en la creación. Porque el mundo es explicitación (explicatio) del máximo absoluto e infinito.

Esta revelación desde la creación y a través de la creación (revelación que es grito en las calles) nos obliga a profundizar. En primer lugar, debemos reconocer que no hay naturaleza sin arte, así como no hay arte sin naturaleza. La naturaleza necesita del artista para explicitar su realidad. La naturaleza es unidad y el arte alteridad, según el De Coniecturis (DC). Ambos se necesitan ${ }^{35}$. Ambos se unen como complicatio-explicatio. La naturaleza en sí es un poder-ser, pero el poder-ser no es (107), aunque sea anterior al existir (130). Por eso, la naturaleza necesita la materia y el artista busca la semejanza, es decir, no quiere hacer una vulgar copia, sino una asimilación de la naturaleza. El artista es un creador, o mejor, un co-creador. Supera la separación entre natural y superficial (cfr. André, 1995: 566-567).

El artista conoce y ayuda a conocer a Dios a través (a partir) de las cosas sensibles. Eso es el sentido profundo del adagio ars imitatur naturam. La naturaleza es primera. El artista no reproduce de manera mecánica, ni siquiera copia según su talento. El arte debe imitar la fuerza generadora de la realidad de la naturaleza, es decir busca imitar el movimiento de la naturaleza y realiza su creatividad a través de una semejanza con esta actividad. El arte es metamorfosis (cfr. André, 1995: 557-558).

En Dios, el querer coincide con el ejecutar (DM 146). Nicolás toma el ejemplo del fabricante de vasos de vidrio: insufla espíritu en el vidrio, el cual ejecuta su voluntad ${ }^{36}$. En Dios, todo es uno. La mente es una cierta fuerza que tiene en sí la imagen del arte divino (DM 148). «La mente ha sido creada por el arte creador como si tal arte hubiese querido

35 «Intelligentiam enim facile ut a ratione emanate divina artem participare concipitur, ut autem a se artem exerit naturam esse videmus. Ars enim imitatio quedam nature existit, alis enim sensibilia naturalia esse, alia artificialia manifestum est, sed non est possibile sensibilia naturalia esse artis expertia ita nec sensibilia artificialia natura cerere possunt» (Se piensa facílmente que la inteligencia participa del arte, en la medida en que emana de la razón divina, pero, puesto que engendra por sí el arte, vemos que es naturaleza. El arte es una cierta imitación de la naturaleza, y es evidente que unas cosas sensibles son naturales, otras son artificiales. Pero no es posible que las cosas sensibles naturales se encuentren privadas de arte, lo mismo que las sensibles artificiales no pueden estar privadas de naturaleza; trad. de J. M. André, 1995: 567).

36 Nicolás profundiza este ejemplo en el De Genesi (DG 163-164). 
crearse a sí mismo» ${ }^{37}$. Sabemos que la imagen nunca será igual al original, pero «la mente tiene una fuerza concreada a través de la cual, cuando está excitada, puede hacer más conforme a la actualidad del arte divino» (DM $149)^{38}$. Así se entiende que el sonido necesite del vidrio para existir y la música de la cítara (DM 150).

Veamos de nuevo las ideas del ignorante sobre su cuchara. La fabricación de una cuchara es un arte y «todo arte humano es una cierta imagen del arte infinito y divino» (DM 59) ${ }^{39}$. Hablar de cierta imagen significa que el arte humano no puede alcanzar una precisión perfecta (DM 60): dos pinturas que parecen representar un mismo objeto, siempre se diferencian (DS II,39). Toda precisión creada es limitada y todo el principiado viene de un principio infinito: su origen es el arte infinito (DM 61).

Todo artista busca acercarse al original para representarlo lo mejor posible. Pero, yo, dice el ignorante:

No imito la figura de cualquier cosa natural. Estas formas de cucharas, copas y ollas se completan por el solo arte humano. Por eso, el arte mío es más consumado que imitador de las figuras creadas y, en eso, es más semejante al arte divino (DM 62) ${ }^{40}$.

No podemos imaginar el arte del ignorante como reproducción (copia) de una cosa sensible como sería mirar una cuchara y esculpir otra igual, mirar una manzana y pintarla. El arte que promueve el ignorante parte de lo no sensible para llegar a una forma sensible (DM 63). Lo no sensible es la «cucharidad» y el artista busca explicitar (hacer explícita) esta esencia de la cuchara en cada cuchara sensible. Por eso hablaba antes (DM 54) de exprimir la cuchara.

El ser humano no puede llegar a la esencia de las cosas y lo sabe. Entonces, ¿locura u orgullo del ignorante?

El cristianismo, al proclamar la fe en un Dios creador, permitió al ser humano buscar y encontrar las huellas (vestigia) del creador en su creación. Esta novedad de la fe cristiana hace posible que el ser humano conozca realmente al Creador, aunque nunca lo pueda conocer en su

37 «Unde mens est creata $\mathrm{ab}$ arte creatice, quasi ars illa se ipsam creare vellet» (trad. J. Machetta).

38 «Sic mens nostra (..) habet tamen vim illam concreatam per quam excitata se actualitati divinae artis conformiorem facere potest».

39 «Omnes humanas artes imagines quasdam esse infinitae et divinae artis».

40 «No imitor figuram cuiuscumque rei naturalis. Tales enim formae cocleares, scutellares et ollares sola humana arte perficiuntur. Unde ars mea est magis perfectiora quam imitatiora figurarum creatarum et in hoc infinitae arti similior». 
esencia. Hace posible también, por el mismo efecto, conocer la creación porque su unidad se genera en el Creador, aunque tampoco conozca su esencia. La filosofía griega separaba irremediablemente el ser divino de los seres creados. El cristianismo los relaciona y permite dar un sentido nuevo a la ciencia y a todo tipo de conocimiento: se puede partir del mundo físico y relacionarlo con la divinidad, se puede empezar con el mundo divino (el Máximo absoluto) y dar cuenta de su presencia e imagen en la creación. Hay un tipo de monismo que excluye todo panteísmo (existe una sola sustancia) y, al mismo tiempo, el mundo y Dios están totalmente separados. Por eso, la sabiduría grita en las calles y el ser humano reconoce su no saber (docta ignorancia) como fuente del saber. Así la ciencia física es posible. El mundo sensible tiene valor pleno (a diferencia de los griegos) y los sentidos son indispensables.

El esfuerzo de Nicolás de Cusa será, sin negar los sentidos, buscar traspasarlos para acercarse a la esencia de las cosas.

Todos los sabios están de acuerdo: lo visible es imagen de lo invisible. Entonces, el Creador puede ser conocido por las creaturas como en un espejo y en enigma. La razón es que todas las cosas tienen relación entre sí. Por eso, se puede investigar a través de símbolos. Esto viene de que el universo es único. Toda imagen, aunque haya semejanza, nunca llega a la imagen tal que no haya otra más semejante y más igual (DDI I, 11,30 ${ }^{41}$.

Nicolás siempre busca los nombres, especialmente el nombre de Dios y, al mismo tiempo, acota que es imposible conocer la esencia de Dos; tampoco de alguna cosa creada (sólo una piedra puede sabe lo que es una piedra). Los nombres surgen a partir de los sentidos nuestros, es decir desde fuera de las cosas nombradas.

¿Hay nominalismo en eso? Creemos que no. Es más: uno de los logros de Nicolás de Cusa es buscar nombres, ver las definiciones de la realidad y de las cosas, y, al mismo tiempo, de darse cuenta y dar cuenta que el arte supera el nominalismo, el arte al estilo cusano. Este esfuerzo empieza en el De Docta Ignorantia y se va perfeccionando en los demás libros. Tomemos el ejemplo de la cuchara. La cuchara es un instrumento de cocina bastante vulgar, quizás tan vulgar como el mismo idiota. Pero

\footnotetext{
41 «Consensere omnes sapientissimi nostri et divinissimi sanctissimique doctores visibilia veraciter invisibilium imagines esse: atque creatorem nostrum ita cognoscibiliter a creaturis videri posse quasi in speculo et in enigmate. Hoc autem quod spiritualia per se a nobis inattingibilia symbolice investigarentur radicem ex his que superius dicta sunt... ut ex omnibus unum exurgat universum... et quamvis omnis imago accedere videatur ad similitudinem exemplari quin per infinitum similior atque equalior esse possit».
} 
este mismo ignorante, consciente de su arte, contempla la madera, hasta encontrar en ella la forma de cuchara. No se trata de mirar las ideas eternas y sacar de ellas la imagen de la cuchara. Para el ignorante (para Nicolás), la cuchara está en el pedazo de madera. Su esfuerzo consiste en verla y exprimirla. Para ver la escultura, dice el Pseudo Dionisio en Teología Mistica, hay que quitar las envolturas. Quitar significa subir hasta los principios. Así resplandece la forma de la cuchara.

Agustín hablaba de illuminatio. H. U. von Balthasar (1986) así la presenta: la ratio busca, el cogito garantiza la verdad, el intelligo comprende. Tenemos la tríada «objeto-facultad-acto que se transforma en ser-vidaespíritu. De allí nace la illuminatio: 'el espíritu finito no puede juzgar sino a la luz del Espíritu absoluto'» (Balthasar, 1986: 110). La illuminatio no es visión, es apertura a la verdad absoluta. «El acto del espíritu va con la facultad estética de percibir en la verdad la cualidad de lo eterno» (Balthasar, 1986: 113). En término del Cusano, podemos decir que el que busca la verdad se eleva hasta llegar al Dios desconocido e invisible.

Entonces, cuando le parece que no hay luz alguna para su razón, con un deseo totalmente entregado, espera que aquel sol omnipotente pueda iluminarlo con su salida que destroza las tinieblas, de manera que vea lo invisible y todo lo que el mismo se le manifieste (De Possest (DP) 15$)^{42}$.

Podemos volver a preguntarnos: ¿Qué es la cuchara? No podemos llegar a la esencia de las cosas. Por eso no podemos nombrarlas. Los nombres son algo impuestos porque se originan en la razón. Y siempre faltará la precisión (DM 58). Sería mejor que estos mismos nombres viniesen de la eternidad (DM 59). Veamos la cuchara. Todo arte es imagen del arte infinito y divino (DM 59). Esto significa que todo arte humano es finito y limitado y que no alcanza la precisión (DM 60). Sólo lo infinito alcanza la precisión; además es sin principio, es decir, eterno (DM 61). Por eso podemos decir que todo arte humano proviene del arte infinito. Tomemos el ejemplo de la cuchara (DM 62): la cuchara tiene su modelo en nuestra mente. El Ignorante no quiere ser como el pintor que busca representar algo. Lo que busca es extraer (educere) la cuchara desde la madera y las ollas y platos desde el barro. No se trata de imitar la naturaleza: el arte humanos es el único en poder realizar, crear sin imitar figuras creadas $y$, en eso, se asemeja al arte divino. ¿Cómo lo hace entonces? (DM 63) Desbasta y cava (ahueca) la madera hasta dar la

42 «Cum nulla luce rationis sue videatur: tunc expectat devotissimo desiderio solem illum omnipotentem, et per suiipsius ortum: pulsata caligine illuminari: ut invisibilem tantum videat quantum seipsum manifestaverit». El párrafo termina con una alusión a $\mathrm{Rm}$ 1,18-20. 
forma, es decir, hasta que resplandezca convenientemente la forma de la «cucharidad» ${ }^{43}$. Tiene entonces una forma que no se puede multiplicar ni comunicar. En todas las cucharas reluce esta «cucharidad», pero nunca con precisión perfecta. Se la llama «cuchara» (DM 64) y este nombre está aceptado porque lo hecho se asemeja a la forma de la cuchara, pero se podría poner otro nombre. El nombre es algo cultural o racional. La razón se ocupa de lo que cae bajo los sentidos y eso es sólo conjeturas. Pero (DM 65) tenemos que admitir que algo no estuvo ni en los sentidos ni en la razón: es decir, el modelo precede lo sensible como la verdad precede la imagen.

La mente es forma sustancial, o sea, la fuerza que tiene todo implícito en sí, a su manera, es decir, que tiene implícita en sí la fuerza animadora por medio de la cual anima el cuerpo, vivificándolo con la vida vegetativa y sensitiva, y que tiene implícita en sí la fuerza raciocinadora intelectual e intelectible (DM 80) ${ }^{44}$.

En cuanto animadora, se llama «alma». ¿El alma existía antes que el cuerpo? (DM 81). Por naturaleza, sí; en el tiempo, no. Así como la vista existe antes que el ojo por naturaleza, pero en acto, sólo está en el ojo.

La mente es una cierta semilla divina que tiene implícita en sí por fuerza propia y nocionalmente los modelos originales (exemplaria) de todas las cosas. Desde Dios del cual la semilla recibe su fuerza y en el momento en que recibe la existencia, esta semilla está localizada simultáneamente en la tierra conveniente (en armonía) donde puede dar fruto y puede explicitar nocionalmente de por sí el conjunto de las cosas ${ }^{45}$.

De lo contrario, existiría en vano. Lo divino es inalcanzable con precisión; por eso, hacemos conjeturas (DM 88). Por ejemplo, la vista: la vista no se ve; sólo siente un obstáculo. El ser humano discierne con el uso de la razón (DM 83). La mente perfecciona la razón (DM 84). La mente tiene juicio porque es imagen del ejemplar de todo ${ }^{46}$, es decir de

43 Este modismo quiere traducir la palabra inventada por el Cusano: coclearitas en DM 64.

44 «Mens est forma substantialis sive vis in se omnia suo modo complicans, vim animativam, per quam corpus animat vivificando vita vegetativa et sensitiva, et vim ratiocinativam et intellectualem et intellectuabilem complicans».

45 «Mens est quoddam divinum semen sua vi complicans omnium rerum exemplaria notionaliter, tunc a Deo, a quo hanc vim habet, eo ipso, quod esse recipit, est simul et in conveniente terra locatum, ubi fructum facere possit et ex se rerum universitatem notionaliter explicare».

46 «Imago exemplaris omnium». 
Dios (DM 85). El ejemplar reluce en la mente como la verdad en la imagen. La imagen duerme hasta que es excitada; es reflejo como la verdad resplandece en la imagen, como el diamante. Si hay cercanía, la conjetura es aceptable (DM 86).

\section{E1 Espejo}

Y el Ignorante toma una hermosa cuchara en su mano para explicar:

Quise hacer una cuchara como espejo; busqué una madera muy compacta y noble por sobre todas; apliqué los instrumentos con cuyo movimiento extraje (elicere) la conveniente proporción, en la que la forma de la cuchara resplandeciera perfectamente. Luego de esto pulí la superficie de la cuchara de tal manera que inserté en el resplandor de la forma de cuchara la forma propia del espejo, como puedes ver. Pues siendo la cuchara perfectamente hermosa, asimismo con este procedimiento es una cuchara que es espejo. Pues tiene en ella todos los géneros de los espejos, es decir, cóncavo, convexo, recto y en columna. Recto en la base del mango, en forma de columna en el mango, en la concavidad de la cuchara cóncavo, en la convexidad convexo. De donde la forma de espejo no tuvo un ser temporal antes de la cuchara, sino que fue por mí añadido a la forma primera de la misma para la perfección de la cuchara, para que la perfeccionara de modo que ahora la forma de espejo contenga en sí la forma de la cuchara. Y la forma de espejo es independiente de la forma de cuchara, pues no es de la esencia del espejo que sea cuchara. Por lo cual, si se quebraran las proporciones sin las cuales no puede haber forma de cuchara, por ejemplo si se separara el mango, dejaría de ser cuchara, pero por ello no dejaría de ser la forma de espejo. De la misma manera, Dios por el movimiento del cielo extrajo (educere) la proporción de la materia adecuada, en la que resplandeciera la animalidad de un modo más perfecto, a la cual después añadió la mente como un espejo viviente según el modo que dije (DM 86-87) ${ }^{47}$.

47 (86) «Volui facere coclear speculare. Quaesivi lignum valde unitum et nobile super omnia. Applicui instrumenta, quorum motu elicui convenientem proportionem, in qua forma coclearis perfecta resplenderet. Post haec perpolivi coclearis superficiem adeo, quod induxi in resplendentiam formae coclearis formam specularem, ut vides. Nam cum sit perpulchrum coclear, est tamen cum hoc coclear speculare». (87) «Habes enim in eo omnia genera speculorum, scilicet concavum, convexum, rectum et columnare: in base manubrii rectum, in manubrio columnare, in concavitate coclearis concavum, in convexitate convexum. Une forma specularis non habuit temporal esse ante coclear, sed ad perfectionem coclearis addita est per me formae primae coclearis, u team perficeret, ut nunc forma specularis in se contineat formam coclearis. Et forma specularis est independans a cocleari. Non est enim de essentia speculi quod sit coclear. Quare si rumperentur proportiones, sino quibus forma coclearis ese nequit, puta si manubrium separareretur, desineret ese coclear, sed ob hoc forma specularis non desineret ese. Ita 
En forma sencilla y solemne, el Ignorante describe la fabricación que es extracción y creación de la cuchara para que descubramos que lo importante no es la cuchara en sí. La cuchara es símbolo de la creación y llega a ser un espejo. Desde el momento de su creación y para siempre, seguirá siendo espejo aún cuando desaparezca como cuchara.

¿Qué es un espejo para Nicolás de Cusa? Para él. El ser humano reflexiona a través de símbolos porque vemos como en espejo y enigma. Está presente la idea de San Pablo: «Ahora vemos en espejo, en enigma. Entonces veremos cara a cara. Ahora conozco de manera parcial, pero entonces conoceré como soy conocido» (1 Co 13,12). Por eso, el Cusano puede insistir: "El entendimiento desea la verdad no a través de enigma o signos, sino apoderarse de ella con certeza y cara a cara» (DDI III,10,241) ${ }^{48}$.

En Pablo, el espejo indica una semejanza-desemejanza entre nuestro conocimiento actual de Dios y la realidad misma. Pero Pablo insiste más en el dinamismo de un conocimiento siempre creciente y, sobre todo, de una anticipación escatológica de este conocimiento pleno. Nicolás sigue la misma senda y entrega su sello personal. La palabra «espejo» y «enigma» hacen referencias al texto paulino; las palabras «símbolo»o «signo» son la traducción filosófica de la misma idea.

Volvamos a la Docta Ignorancia. En la primera parte, Nicolás afirma la unidad de la creación y del Creador proclamando que:

Las cosas visibles son verdaderamente imágenes de las invisibles y el Creador puede ser visto y reconocido por las creaturas como en un espejo y en enigma. El hecho que se pueda investigar de modo simbólico las cosas espirituales - para nosotros inalcanzables en sí - tiene su raíz en que todas las cosas tienen entre sí una cierta proporción - escondida para nosotros e incomprensible - de tal manera que surja un único conjunto y que todas sean una unicidad con el único máximo (DDI I,11,30) ${ }^{49}$.

El Cusano expresa las mismas ideas que San Pablo (1 Co 13,12): no podemos conocer la realidad profunda de las cosas porque sólo vemos a

quidem Deus per motum caeli de apta materia proportionem eduxit, in qua resplenderet animalitas perfectiori modo, cui deinde addidit mentem quasi vivum speculum modo auo dixi» (trad. de J. Machetta).

48 «...cum ultimo desiderio non aliud desideret quam ipsam veritatem non in enigmate aut signis: sed certitudinaliter facietenus apprehendere».

49 «Visibilia veraciter invisibilium imagines esse, atque creatorem nostrum ita cognoscibiliter a creaturis videri posse quasi in speculo et in enigmate. Hoc autem quod spiritualia per se a nobis inattingibilia symbolice investigarentur radicem... quoniam omnia ad se invicem quandam nobis tamen occultum et incomprehensibilem habent proportionem ut ex omnibus unum exurgat universum, et omnia in uno maximo sint ipsum unum» (trad. personal con la ayuda de J. Machetta). 
través de un espejo. En otro libro anterior al De Mente, el De Filiatione Dei (DFD), Nicolás especificó su idea. No podemos entender plenamente la verdad, no podemos verla. Sólo la alcanzamos a través de un espejo. Y no sólo a través de un espejo único, sino que a través de varios espejos cada vez más defectuosos (DFD 67). El espejo de la verdad es un espejo recto y es el espejo más verdadero, porque muestra la verdad en su esplendor. Más intelectual es la visión y más se acerca a este espejo de la verdad «en el cual todas las cosas resplandecen en verdad, tal como son, sin defectos» (DFD 67) ${ }^{50}$. El espejo intelectual es algo vivo, es decir, no es pura intelectualidad. Y este espejo vivo intelectual «recibe en sí aquel rayo transparente (specular) del espejo de la verdad, es decir, el que tiene en sí la verdad de todos los espejos» (DFD 67) ${ }^{51}$. Lo mismo pasa con el ojo: porque es vivo, se contempla tal como es y también todas las demás cosas.

Cuanto más simple, absoluto, claro, limpio, recto, justo y verdadero sea el espejo y de manera más limpia, gozosa y verdadera contemplará en sí la gloria de Dios y todas las cosas. Este primer espejo de la verdad, podemos llamarlo verbo, logos o Hijo de Dios. En él, el espejo intelectual alcanza la filiación, de tal manera que sea todo en todos y todo en él, y que su reino sea posesión de Dios y de todo en la vida gloriosa (DFD 67) ${ }^{52}$.

Nicolás lee el texto de la Primera Carta a los Corintios en clave cristológica. Vemos en espejo: esa es la diversidad de la creación. Pero tenemos un primer espejo que nos muestra la realidad con mayor nitidez: Cristo, como imagen del Dios invisible (Col 1,15), es el espejo primero. Por eso, todo tiene en él su consistencia (Col 1,17). En Cristo, Dios es todo en todos (1 Co 15,28).

En el De Beryllo (DB), escribe el Cusano:

Quien lee lo que escribí en varios libros, verá que me vuelvo muy seguido en la coincidencia de los opuestos y que me esforcé con frecuencia de encerrar junto con la visión intelectual que excede el vigor de la razón. De allí sacaré claramente un concepto para el lector: levantaré el espejo y

\footnotetext{
50 «Speculum primum veritatis rectum, in quo veraciter omnia uti sunt absque defectu resplanderet».

51 «...in se recipit specularem illum radium speculi veritatis: in se habentis ómnium speculorum veritatem».

52 «Quanto enim simplicius absdolutius clarius mundius rectius iustius et verius fuerit: tanto in se gloriam Dei atque omnia limpidius gaudiosius veriusque intuebitur. In speculo igitur illo primo veritatis quod et verbum logos seu flius dici potest adipiscitur intellectuale speculum filiationem: ut sit omnia in omnibus et omnia in ipso: et regnum eius possessio Dei et omnium in vita gloriosa». Cfr. D'Amico, 2005: 24).
} 
enigma por el cual el débil de cualquier inteligencia de lo posible de saber se ayuda y dirige en lo último y pondré las cosas difíciles de los sabios y las pocas sentencias y opiniones como las difíciles como en un espejo firme y en enigma por la visión intelectual (DB 1) ${ }^{53}$.

El hombre es un segundo dios. En efecto, como Dios es creador de los entes reales y de las formas naturales, así el hombre es creador de los entes racionales, formales y artificiales que no son sino semejanzas de su intelecto, como las creaturas son semejanza del intelecto divino de Dios. Por eso, el hombre tiene intelecto que es semejanza del intelecto divino cuando crea (in creando). El hombre crea las semejanzas de las semejanzas del intelecto divino como son las figuras extrínsecas, las formas naturales de la forma intrínseca, donde mide su intelecto por el poder de sus obras y, de allí, mide el intelecto divino como la verdad es medida a través de la imagen. Esa es la ciencia enigmática. Sin embargo tiene la vista muy sutil por la cual ve que el enigma es enigma de la verdad, de tal modo que sabe que esta verdad existe y no está en figura en algún enigma (DB 7) ${ }^{54}$.

Volvamos al De Mente. El ignorante no presenta la cuchara a través de un espejo, sino que la cuchara es un espejo y la manera de construirla, de extraerla o exprimirla refleja la búsqueda de un espejo lo más perfecto posible. El artista busca acercarse al primer espejo. Su trabajo -una simple cuchara- debe reflejar la verdad de la creación y de Dios. Un hombre despreciable con un trabajo muy poco apreciado puede, quiere y debe elevarse donde no llega el filósofo con todos sus estudios y su arrogancia. La creación de una «simple» cuchara es literalmente el reflejo de la creación en Cristo.

Última mención de la cuchara, siempre adosada a un tema preferencial del Cusano: lo múltiple y lo grande. La multiplicidad va con

\footnotetext{
53 «Qui legerit ea quae in variis scripsi libellis videbit me in oppositorum coincidentia crebrius versatum, quodque nisus sum frequenter iuxta intellectualem visionem que excedit rationis vigorem concludere. Unde ut quam clare legenti conceptum depromam: speculum et enigma subiiciam, quo se infirmus cuiusque intellectus in ultimo scibilium iuvet et dirigat, et graviores doctissimorum in difficilibus ponam paucas sententias et opiniones, ut applicato speculo et enigmate».

54 «Hominem esse secundum deum. Nam sicut deus est creator entium realium et naturalium formarum, ita homo rationalium entium et formalium artificialium que non sunt nisi sui intellectus similitudines, ideo homo habet intellectum qui est similudo divine intellectus in creando. Hinc creat similitudines similitudinum divini intellectus : sicut sunt extrinsece artificiales figure, similitudines intrinsece naturales forme, unde mensurat suum intellectum per potentiam operum suorum, et ex hoc mensurat divinum intellectum, sicut veritas mensuratur per imaginem, et hec est enigmatica scientia. Habet autem visum subtilissimum per quem videt enigma esse veritatis enigma ut sciat hanc esse veritatem que non est figurabilis in aliquo enigmate».
} 
distinción; la magnitud con integridad (DM 126). Para saber una parte de algo, hay que saber el todo (DM 127). Para conocer a los hombres, hay que conocer la humanidad. Para extraer (exscindere) la cuchara por parte, hay que mirar la totalidad y así extraer (elucere) una cuchara bien proporcionada.

Toda la cuchara que concebí en la mente, es el modelo original hacia el cual dirijo la mirada mientras formo (fingere) la parte ${ }^{55}$.

Sólo

...entonces se puede fabricar una cuchara perfecta porque cada una de las partes conserva su proporción en orden al conjunto ${ }^{56}$.

De allí podemos entender que Dios es el modelo original de la universalidad. Ignorar a Dios es ignorar la universalidad y entonces desconocer las partes del conjunto. Acoger a Dios, es entrar en la universalidad y conocer cada una de las partes.

\section{Conclusión}

Tenemos que concluir. El ignorante, a través de arte y de su manera de explicar su arte, se transforma en el maestro. La Docta Ignorancia está siempre presente y vigente. Nicolás de Cusa nos revela su arte de la conversación y su manera pausada y continua de profundizar un tema. La cuchara es la creación. Fue creada por arte, no sacando de unas ideas, sino que fue exprimida. Así crea Dios al ser humano del barro. El ser le es dado por aquel que da el ser y, por ende, es el ser y es el ser máximo. $\mathrm{El}$ artista no es sólo aquel que plasma o representa algo, es realmente un creador, o mejor, un co-creador.

La mente no mira las ideas platónicas, pero sí mira el espejo y el espejo primero es Cristo. El arte es entonces cristológico y porque es cristológico es sacramental: en Cristo, podemos ver lo visible y referirnos a lo invisible; en Cristo, podemos 'exprimir' los sacramentos desde la materia.

La cuchara es el ser creado: está en la mente de Dios y está en la materia. Todo arte puede ser perfeccionado. Pero «nuestra mente tiene

\footnotetext{
55 «Totum cochlear, quod mente concepi, est exemplar, ad quod respicio, dum partem fingo».

56 «Tunc possum perfectum cochlear efficere, quando quaelibet pars proportionem suam in ordine ad totum reservat».
} 
una fuerza co-creada a través de la cual, si es excitada, puede hacerse más conforme a la actualización del arte divino» (DM 149) ${ }^{57}$. Al crear la cuchara, el Ignorante hace actual el arte de Dios y une el cielo y la tierra. Para crearla (extraerla), tuvo que mirar al Creador y mirar la madera. Al contemplar la madera, pudo conocerla más profundamente y entonces expresar y exprimir su contenido. El Ignorante es co-creador, no un imitador y menos un repetidor. Hace los gestos de Dios.

Concluyamos con una defensa apologética del Cusano:

Cuando nombramos a Dios Creador, decimos que Él es y nos elevamos hacia la coincidencia y decimos que Dios coincide con el ser. Moisés lo nombró 'formador' (Dios formó al hombre). Si Él es la forma de las formas, el mismo da el ser aunque la forma de la tierra da el ser a la tierra y la forma del fuego al fuego. Pero la forma que da el ser es Dios, el que forma toda forma. Entonces así como la imagen tiene la forma que le da el ser por el cual es imagen, y la forma de la imagen es forma formada y eso que es de la verdad, no tiene sino de la forma que es verdad y modelo original, así toda creatura es en Dios lo que es. En efecto allí existe toda creatura que es imagen de Dios en su verdad (Apologia Doctae Ignorantiae (ADI) 37$)^{58}$.

El Ignorante con su arte busca la forma y busca el que da la forma. Encuentra en su esfuerzo a aquél que da el ser y la cuchara.

\section{REFERENCIAS}

-André, J. M. (1995). La dimensión simbólica del arte en Nicolás de Cusa. Anuario Filosófico, 28 (3), 547-582.

-Balthasar, H. U. von (1986). La Gloria (J. L. Albizú, Trad., T. II). Madrid: Encuentro.

-Cusa, N. de (1999). Un ignorante discurre acerca de la sabiduría (J. Machetta y C. D’Amico, Trads.). Buenos Aires: Eudeba.

\footnotetext{
57 «Mens nostra... habet tamen vim illam concreatam per quam excitata se actualitate divine artis conformiorem facere potest».

58 «Cum creatorem nominemus Deum: et dicimus eum esse: ad coincidentiam nos elevantes dicimus Deum cum esse coincidere. Moyses nominat eum formatorem, formavit igitur Deus hominem, etc. (Gn 2,7), si igitur ipse est formarum forma: ipse dat esse, licet forma terre det terre esse, et forma ignis igni. Forma vero que dat esse Deus est qui format omnem formam. Unde sicut ymago habet formam que dat ei esse hoc per quod est ymago, et forma ymaginis est forma formata, et id quod est veritatis non habet nisi ex forma que est veritas et exemplar, sic omnia creatura in Deo est id quod est, nam ibi est omnis creatura que est ymago Dei in sua veritate».
} 
-Cusa, N. de (2003). Acerca de la docta ignorancia. (Segunda Edición). (J. Machetta y C. D’Amico, Trads., T. I). Buenos Aires: Biblos.

-Cusa, N. de (2005). Un ignorante discurre acerca de la mente. (J. Machetta, Trad.), Buenos Aires: Biblos.

-Cusa, N. de (2009). Acerca de la docta ignorancia. (Segunda Edición, J. Machetta y C. D’Amico, S. Manzo, Trads., T. II). Buenos Aires: Biblos.

-Cusa, N. de (2009). Acerca de la docta ignorancia. (J. Machetta y E. Ludueña, Trads., T. III). Buenos Aires: Biblos.

-D’Amico, C. (2005). Introducción. En N. de Cusa, Un ignorante discurre acerca de la mente (J. Machetta, Trad., págs. 22-27). Buenos Aires: Biblos.

-González Ríos, J. (2005). Presentación de los personajes. En N. de Cusa, Un ignorante discurre acerca de la mente (J. Machetta, Trad., págs. 149-155). Buenos Aires, Biblos.

-Gress, Th. (2010). Nicolás de Cues: la sagesse selon l'idiot. Recuperado el 01 de Diciembre de 2010: http://www.actu-philosophica.com.

-Kues, N. von (1967). Werke (P. Wilpert, Ed.; Band 1-2). Berlín: de Gruyter.

-Matteoli, A. (2010). "Idiota», ausencia de cultura u opción por la privacidad. Recuperado el 01 de Diciembre de 2010: http://www.ofs-rgbl.com.ar.

-Rienzo, R. di (2005). La mente: viva imagen de Dios. En N. de Cusa, Un ignorante discurre acerca de la mente (J. Machetta, Trad., págs. 163-170). Buenos Aires: Biblos.

-Silva, J. (2010). La identidad laical. Reflexiones a partir del Documento de Aparecida. La Revista Católica, CIX (1.164), 287-292.

Sumario: I. Los actores; 1. El De Mente; 2. El Idiota; II. La cuchara; 1. El encuentro; 2. El arte; 3. El espejo; Conclusión; Referencias. 